\title{
Ixekizumab for Patients with Plaque Psoriasis Affected by Multiple Sclerosis Case report
}

Enrique Herrera-Acosta, "Gustavo G. Garriga-Martina, Jorge A. Suárez-Pérez, Eliseo A. Martínez-García, Enrique Herrera-Ceballos

ABSTRACT: Multiple sclerosis (MS) is an autoimmune demyelinating disorder of the central nervous system that shares similar immunopathogenic mechanisms with chronic plaque psoriasis, such as the overexpression of the Th17 pathway. We report a 50-year-old male patient with MS and severe chronic plaque psoriasis who presented to Hospital Virgen de la Victoria, Málaga, Spain, in 2019. He was successfully treated with ixekizumab (anti-interleukin [IL]-17A and IL-17A/F monoclonal antibody). The treatment achieved complete skin clearance (i.e. a Psoriasis Area Severity Index 100 response) with no adverse event and no evidence of progression of the neurological disease either.

Keywords: Psoriasis; Ixekizumab; Multiple Sclerosis; Case Report; Spain.

$\mathrm{M}$ ULTIPLE SCLEROSIS (MS) IS AN AUTOimmune demyelinating disorder of the central nervous system. MS shares significant immunopathogenic mechanisms with psoriasis such as the overexpression of Th17 lymphocytes and associated cytokines. ${ }^{1,2}$ We report the case of a patient presenting with MS and severe psoriasis who was successfully treated with ixekizumab (anti-IL-17A and IL-17A/F monoclonal antibody). To the best of the authors' knowledge, this is the first case of its kind to be reported in the medical literature.

\section{Case Report}

We report a 50-year-old male patient with a 40-year history of chronic plaque psoriasis who presented to Hospital Virgen de la Victoria, Málaga, Spain, in 2019. He was diagnosed with this in 1979 along with primarily progressive MS which made the patient dependent on others for his basic daily activities. His usual medications included muscle relaxants such as baclofen (25 mg every $12 \mathrm{~h}$ ) and tizanidine hydrochloride (2 mg every $12 \mathrm{~h}$ ).

His psoriasis had been initially treated with methotrexate and acitretin and then switched to ustekinumab (45 mg) due to the inefficacy of the former. However, a Psoriasis Area Severity Index (PASI) of 14 persisted after increasing the dose of ustekinumab to $90 \mathrm{mg}$ every eight weeks [Figure 1].

Due to the MS, for which the use of anti-tumor necrosis factor (TNF) alpha biological agents is not indicated, the treatment was changed to include ixekizumab (80 mg), following the dosage instructions provided in the drug's datasheet. After four weeks, the patient presented with a PASI of 3 [Figure 2].

During the subsequent follow-ups, the patient achieved complete skin clearance (PASI 100 response) and this is maintained at the time of writing this report, i.e. three years after the initiation of the ixekizumab (80 mg) treatment. Moreover, no adverse events occurred during this follow-up period and laboratory investigations were normal. As for MS, the disease remains stable with no new symptoms or signs of progression after starting the ixekizumab treatment.

The patient signed a written consent to permit the publishing of his clinical data and photographs.

\section{Discussion}

Pathogenic mechanisms of MS have not yet been sufficiently understood. An autoimmune aetiology must be considered, but genetic and environmental factors may play a role. Regarding these immune factors, the roles played by $\mathrm{T}$ helper Type 1 (Th1), Th17, CD8+ T cells and macrophages stand out as they attack the proteins that form the myelin sheaths of the central nervous system. This provides the needed scope for a connection being established between psoriasis and MS as they share, at least partially, similar immunopathogenic mechanisms such as the overexpression of the Th17 pathway. The evidence concerning this is strong as multiple studies have pointed to the involvement of Th17 lymphocytes and their cytokines in the immunopathogenesis of MS. ${ }^{2-4}$ 


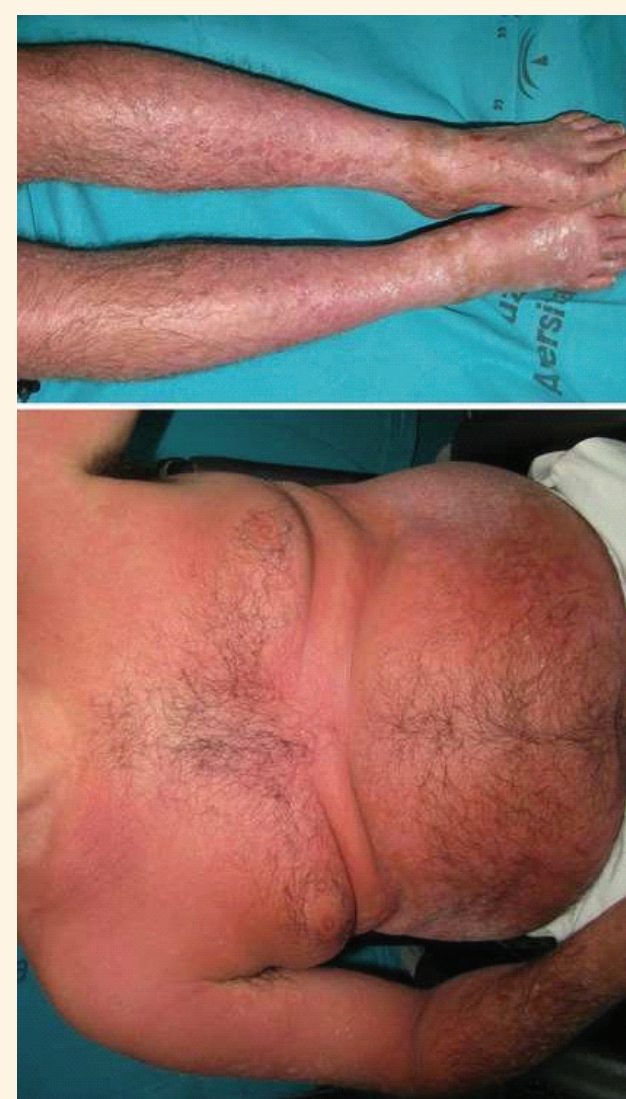

Figure 1: Baseline photographs of the chest and legs of a 50-year-old male patient with multiple sclerosis and plaque psoriasis at the time of starting treatment with ixekizumab.

Recently, Li et al. published a meta-analysis, wherein they found an increase in the proportion of peripheral blood Th17 cells and IL-17 and IL-23 levels in patients with MS when compared with healthy subjects. ${ }^{2}$

This fact, together with the increased risk of incident psoriasis in patients with MS (amounting to a $54 \%$ higher risk than in the healthy population), or the existence of drugs approved for both diseases, such as fumarate, suggests that there is a degree of overlap in the immunopathogenic mechanisms of both these diseases. ${ }^{5,6}$

However, there are also major differences, as the family of anti-TNF alpha drugs, used in cases of severe psoriasis, is not indicated for patients with demyelinating diseases, including MS, where an exacerbation or recurrence of neurological symptoms has been observed in rare cases.,8 In contrast, biological anti-interleukin drugs, such as ustekinumab (anti-IL-12/23) or secukinumab, have been successful in patients with psoriasis and MS; these drugs have shown no negative effect on the natural history of the neurological disease..$^{9-11}$

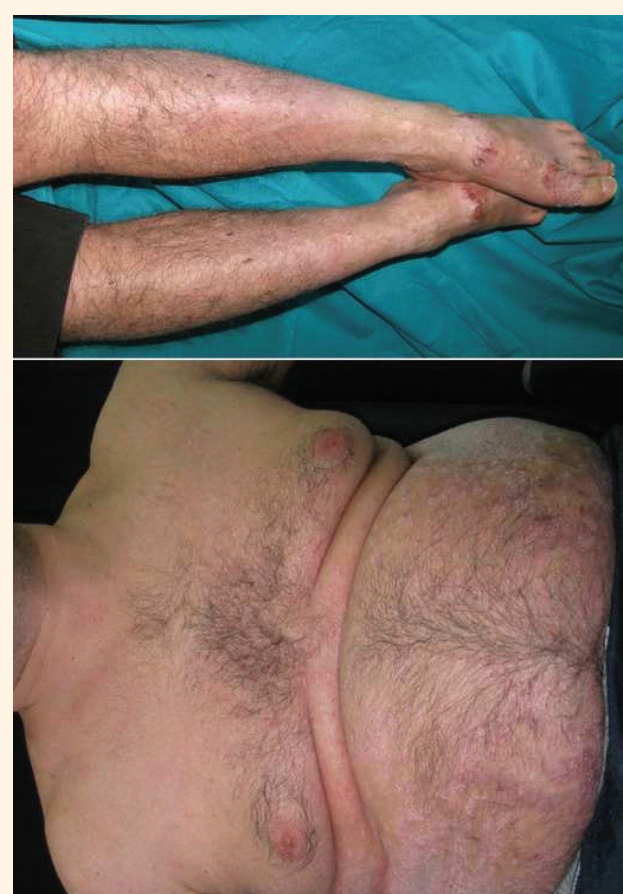

Figure 2: Photographs of the chest and legs of a 50-yearold male patient with multiple sclerosis and plaque psoriasis four weeks after the start of treatment with ixekizumab.

\section{Conclusion}

This report presented a patient with MS and severe chronic plaque psoriasis who was successfully treated with ixekizumab. This treatment has proven to be highly effective, bringing about complete skin clearance (PASI 100 response) and safe as there was no evidence of progression of the neurological disease or any adverse event after this treatment.

\section{AUTHORS' CONTRIBUTION}

All authors took part in the clinical management of the patient and drafting of the manuscript. All authors approved the final version of this manuscript.

\section{References}

1. Katsara M, Apostolopoulos V. Editorial: Multiple sclerosis: Pathogenesis and therapeutics. Med Chem 2018; 14:104-5. https:// doi.org/10.2174/157340641402180206092504.

2. Li YF, Zhang SX, Ma XW, Xue YL, Gao C, Li XY. Levels of peripheral Th17 cells and serum Th17-related cytokines in patients with multiple sclerosis: A meta-analysis. Mult Scler Relat Disord 2017; 18:20-25. https://doi.org/10.1016/j.msard.2017.09.003.

3. Moser T, Akgün K, Proschmann U, Sellner J, Ziemssen T. The role of TH17 cells in multiple sclerosis: Therapeutic implications. Autoimmun Rev 2020; 19:102647. https://doi.org/10.10 16/j.autrev.2020.102647. 
4. Bedoya SK, Lam B, Lau K, Larkin J. Th17 cells in immunity and autoimmunity. Clin Dev Immunol 2013; 2013:986789. https:// doi.org/10.1155/2013/986789.

5. Guido N, Cices A, Ibler E, Huynh T, Majewski S, Sable K, et al. Multiple sclerosis association with psoriasis: A large U.S. population, single centre, retrospective cross-sectional study. J Eur Acad Dermatol Venereol 2017; 31:e397-8. https://doi.org/10.11 11/jdv.14205.

6. Marrie RA, Patten SB, Tremlett H, Wolfson C, Leung S, Fisk JD. Increased incidence and prevalence of psoriasis in multiple sclerosis. Mult Scler Relat Disord 2017; 13:81-6. https://doi. org/10.1016/j.msard.2017.02.012.

7. Fernández-Espartero MC, Pérez-Zafrilla B, Naranjo A, Esteban C, Ortiz AM, Gómez-Reino JJ, et al. Demyelinating disease in patients treated with TNF antagonists in rheumatology: Data from BIOBADASER, a pharmacovigilance database, and a systematic review. Semin Arthritis Rheum 2011; 41:524-33. https://doi. org/10.1016/j.semarthrit.2010.06.004.
8. Andreadou E, Kemanetzoglou E, Brokalaki C, Evangelopoulos ME, Kilidireas C, Rombos A, et al. Demyelinating disease following anti-TNF treatment: A causal or coincidental association? Report of four cases and review the literature. Case Rep Neurol Med 2013; 2013:671935. https://doi.org/10.1155/2013/671935.

9. Venturini M, Zanca A, Venturuzzo A, Filippini M, Frassi M, Tincani A, et al. Secukinumab for patients with plaque psoriasis affected by multiple sclerosis: A mini-review with a represent 0 ative case report. J Eur Acad Dermatol Venereol 2020; 34:e110-12. https://doi.org/10.1111/jdv.16035.

10. Assefa GT, Kaneko S, Oguro H, Morita E. Treatment of psoriasis and psoriatic arthritis with secukinumab after unsatisfactory response to ustekinumab in multiple sclerosis patient. J Dermatol 2019; 46:e112-13. https://doi.org/10.1111/1346-8138.14619.

11. Chang S, Chambers CJ, Liu FT, Armstrong AW. Successful treatment of psoriasis with ustekinumab in patients with multiple sclerosis. Dermatol Online J 2015; 21:13030/qt3bs971cr. https:// doi.org/10.5070/D3217028112. 